\title{
What next? Managing lymph nodes in men with penile cancer
}

\author{
Michael Leveridge, $M D{ }^{*} D$. Robert Siemens, $M D ;^{*}$ Christopher Morash, $M D^{\dagger}$
}

\section{Abstract}

The management of patients with squamous cell carcinoma of the penis is often daunting given its rarity and subsequent lack of high-level evidence to support our decision-making. This culminates in the complex surgical issues involving the management of the regional lymph nodes, which is of critical importance to both quantity and quality of life for these patients. This review aims to highlight the decisive issues surrounding the management of the pelvic and inguinal lymph nodes in the setting of squamous cell carcinoma of the penis, and to spotlight recently published information that adds credence to accepted management strategies of both the clinically positive and negative groin after successful management of the primary lesion.

CUAJ 2008;2(5):525-31

$\mathrm{P}$ enile cancer remains an uncommon and potentially complex urological diagnosis in our practice in Canada. Although management of the primary lesion in itself is often problematic, any discussion or consideration of prognosis in patients with squamous cell carcinoma of the penis must also involve delineation of the presence and extent of metastatic disease to regional lymph nodes. Success in the management of penile cancer relies on the timely and appropriate management of the inguinal and pelvic lymph nodes. ${ }^{1-3}$ This review aims to highlight the issues surrounding the management of the inguinal and pelvic lymph nodes in the setting of squamous cell carcinoma of the penis and to spotlight recently published information that adds credence to accepted management strategies or that approaches nodal disease in a novel or previously unexplored manner.

In this review we will first focus on the management of the clinically positive groin, including lymph node dissection technique, as well as the management of pelvic lymph nodes and the role of chemotherapy in the neoadjuvant, adjuvant or palliative setting. Consideration will also be given to the management of the contralateral groin in unilaterally clinically positive disease. Many of the concepts presented are critical in describing and appreciating the often more complex clinical scenario of the patient with a clinically negative examination after successful management of the primary penile cancer. In highlighting the clinically negative groin, we discuss risk stratification based on the pathological characteristics of the primary lesion and the role of sentinel node biopsy and other methods of identifying occult metastasis.

\section{Palpable inguinal lymph nodes}

Lymphatic drainage of the penis follows a well-recognized and estab- lished pattern of sequential drainage to nodes in the superficial (above the fascia lata) and deep (superior and medial to the saphenofemoral junction beneath the fascia lata) inguinal regions and subsequently to pelvic lymph nodes associated with the ipsilateral iliac vessels and obturator fossa. ${ }^{4-6}$ Presentation with visceral metastatic disease is rare. ${ }^{6}$ Drainage to one or both sides is possible and has been shown to be reproducibly demonstrable with lymphoscintigraphy in the same patient at different times. ${ }^{4,7,8}$

Recent series have demonstrated rates of clinical node positivity at presentation between $22 \%$ and $61 \% .^{5,9,10}$ Lymphadenopathy in patients with clinically palpable nodal disease should be reconciled between reactive adenopathy and true metastatic disease, as infection of this cutaneous neoplasm can also enlarge nodes in the groin. False-positive rates for palpable adenopathy can exceed $50 \%{ }^{3,7}$ A 6-week course of antibiotics can be used to treat and resolve reactive nodes, raising the specificity of palpable adenopathy to $70 \%-86 \%$. ${ }^{3,11}$ Others have advocated for the routine use of fine-needle aspiration of such nodes instead of antibiotic trial therapy, with false-negative rates of $15 \%$. $^{7}$ Patients with persistent palpable lymphadenopathy are candidates for inguinal lymph node dissection in the absence of confirmed visceral metastatic disease or a local burden of matted and unresectable nodes.

\section{Inguinal lymph node dissection}

The classic radical groin dissection involves dissection of superficial and deep inguinal nodes in a quadrilateral bounded by the inguinal ligament superiorly, a line extending $15 \mathrm{~cm}$ down from the pubic tubercle medially, a line extending $20 \mathrm{~cm}$ down from the anterior superior iliac spine laterally and a line connecting the inferior ends of the 
Leveridge et al.

lateral and medial lines. ${ }^{12} \mathrm{~A}$ modification of the radical inguinal lymphadenectomy involves removal of superficial and deep nodes within the triangle bounded by the inguinal ligament, the sartorius muscle laterally and the adductor longus muscle medially, with skin flaps containing all tissue above the Scarpa fascia, and sparing of the saphenous vein and its branches..$^{1,2,13}$ Catalona $^{14}$ pioneered a well-adopted modification that shortens the skin incision, limiting the lateral extent of the node dissection to the femoral vessels. Radical dissection remains the procedure of choice for cases of documented nodal metastases to the groin.

Morbidity has long been of great concern with radical groin dissection and is reported in up to $57 \%$ of groins in $39 \%$ of patients. ${ }^{13}$ Radical inguinal lymphadenectomy has demonstrated increased morbidity as compared with modified approaches. ${ }^{15}$ Common complications include lymphedema, lymphocele or seroma formation, skin flap necrosis and wound infection. ${ }^{13,15,16}$ These can be minor or can result in significant morbidity and the need for secondary procedures for management. Death has been rarely reported in some series, usually as a result of sepsis or from deep vein thrombosis and subsequent pulmonary embolism. ${ }^{3,13}$ Strategies to minimize morbidity include aggressive wound care, minimal intraoperative flap handling, preservation of robust subcutaneous tissue, early ambulation (or, conversely, bed rest in some centres), use of compression stockings, wound drains, antibiotic coverage and appropriate use of myocutaneous flap coverage. ${ }^{13,16}$ A recent publication highlights efforts to undertake endoscopic inguinal lymphadenectomy. ${ }^{17}$

\section{Burden of inguinal disease}

It has been clearly shown that early lymphadenectomy for clinically occult metastases carries a significant survival benefit in penile cancer patients, as will be discussed later in this review. This follows work that has demonstrated that the extent of metastatic disease in the nodes has great prognostic implications., ${ }^{3,5}$ Series have shown 5-year mortality rates of roughly $25 \%$ for low-volume inguinal metastases and $75 \%$ for patients with greater than 2 positive nodes. ${ }^{3,6} \mathrm{~A}$ recent large retrospective review of more than 100 patients undergoing lymphadenectomy for penile cancer demonstrated that disease in more than 2 lymph nodes, extracapsular nodal disease, bilateral node-positive disease and pelvic lymph node metastasis are independent prognostic factors for disease-specific survival. ${ }^{3}$ One of $16(6 \%)$ patients among those with 1-2 positive nodes and no extracapsular spread died of penile cancer, and 42 of 80 patients (53\%) with more extensive adenopathy died of their disease at a median follow-up of 85 months. The authors have proposed a modification to the node component of the TNM (tumour, node, metastases) staging to reflect these findings.

\section{Pelvic lymph nodes}

Disease in pelvic lymph nodes carries a particularly grave prognosis, with 5-year survival rates of less than $20 \% .^{2,6}$ In a series by Lont and colleagues, ${ }^{5}$ 25 of 102 patients $(25 \%)$ with pathologically proven inguinal metastases were later proven to have pelvic node disease, and 4 of these patients (16\%) achieved long-term disease-free survival. Risk factors for the presence of pelvic nodal disease in patients with inguinal node disease included more than 2 positive nodes, extracapsular extension of disease and presence of high-grade cancer in inguinal nodes. These figures are in keeping with several previously published results and many authors have suggested that pelvic lymphadenectomy may be deferred in patients without any of these negative prognostic factors. ${ }^{2,3,5,12}$ However, prospective data are lacking with respect to this assertion, and it is apparent from the literature that a pelvic lymphadenectomy is a common practice in managing patients with penile cancer. ${ }^{5,16}$

\section{Chemotherapy in penile cancer}

Chemotherapy has been employed in patients with squamous cell carcinoma of the penis with mixed success. Indications have ranged from neoadjuvant therapy in advance of surgical resection, to adjuvant therapy for extranodal or pelvic nodal disease, to palliation of advanced cancer. ${ }^{3,6}$ In each case there is a dearth of randomized, prospective data owing to the low incidence of advanced penile cancer. Single-agent chemotherapy with bleomycin, methotrexate or cisplatin has resulted in partial response rates of up to $50 \%$, but with a duration of less than 6 months. ${ }^{6}$ Combination chemotherapy has also been assessed, perhaps most notably in a prospective trial of bleomycin, 
methotrexate and cisplatin. ${ }^{18}$ This demonstrated a $33 \%$ response rate, but a significant burden of toxicity (including $11 \%$ treatment-related death). Vincristine, bleomycin and methotrexate have shown a $54 \%$ response rate in patients with unresectable inguinal nodal disease. ${ }^{6}$ A recent phase II study assessed irinotecan and cisplatin in patients with locally advanced penile cancer. ${ }^{19}$ Response rates were $29 \%$ and $32 \%$ in the neoadjuvant and advanced-disease settings, respectively. Duration of response and survival were not assessed.

Two recent studies have demonstrated the efficacy of neoadjuvant chemotherapy in the setting of matted or unresectable nodal disease in advance of surgical treatment. ${ }^{20,21}$ Numerous regimens with variable cycles were employed in these retrospective studies, most commonly ifosfamide, paclitaxel and cisplatin, and bleomycin, methotrexate and cisplatin. Response rates were $50 \%$ and $63 \%$ in these series, with 5 -year survival in responders at $40 \%$ and $56 \%$. Only 1 nonresponder was alive at 5 years, with the others universally succumbing to their disease within 1 year. Burden of nodal disease on surgical consolidation was associated with survival in the study by Bermejo and colleagues, ${ }^{21}$ as all patients with more than 3 positive nodes died, with a median survival of 23 months (v. 48 mo for those with $<3$ positive nodes).

\section{The contralateral groin}

A significant issue in risk stratification and management in patients with carcinoma of the penis surrounds the management of the contralateral groin in patients with unilateral disease. Little recent research has addressed this issue, but the general consensus seems to involve managing the contralateral groin based on the timing and extent of disease in the ipsilateral groin. ${ }^{1}$ In patients presenting with a high-risk primary tumour or an intermediate-risk lesion with other adverse prognostic factors (lymphatic and vascular invasion, infiltrating pattern of invasion), a bilateral dissection is indicated because of the known bilateral nature of penile lymphatic drainage. In the event of delayed unilateral adenopathy, the contralateral groin is at substantially less risk of harbouring metastatic disease and full dissection may not be warranted, assuming metastases to each groin would have a similar growth rate. ${ }^{1}$ What exactly constitutes delayed presentation of disease is not well described in the literature. It may be reasonable to suggest that a delayed presentation occurs when a groin that is clinically negative at presentation subsequently becomes clinically positive on repeat assessment. Kroon and colleagues ${ }^{22}$ defined delayed lymphadenectomy as one "performed at first sign of lymph node involvement in the course of a watchful waiting program." Recommendations for surveillance protocols after management of the primary tumour call for groin examinations every 2 months for 2 years, every 3 months for the next year and every 6 months until year 5. ${ }^{1}$ Dissection of the contralateral groin is indicated in the late-recurrence patient if the clinically positive side has more than 1 node involved with cancer, as the incidence of contralateral disease rises from about $10 \%$ to $30 \%{ }^{1,2}$

\section{Clinically negative inguinal lymph nodes}

The clinically negative groin has been of particular concern in patients with penile carcinoma. Up to $30 \%$ will harbour occult metastasis that can be uncovered at lymphadenectomy or can present as palpable adenopathy at a later time.,2, The concern lies in risking the unnecessary morbidity of inguinal lymph node dissection in $70 \%$ or more of these patients, or the obverse problem of allowing delayed presentation of metastatic disease. This raises several points of contention in the management of penile cancer, namely, limiting the morbidity of lymph node dissection (without sacrificing its sensitivity in detecting metastases) in patients and the population at risk, increasing the detection of (or confirming the absence of) metastatic deposits in clinically negative groins and optimizing the timing of lymphadenectomy in penile cancer patients.

With such a burden of morbidity relating to node dissection, there has long been an impetus and effort to identify those among patients with clinically negative groins who will not harbour metastatic disease and thus would not benefit from lymphadenectomy. Strategies have included specialized imaging techniques, less extensive surgical procedures and surveillance of patients for delayed development of clinically positive groins. High-resolution ultrasonography has been used to identify changes in lymph nodes that indicate infiltration with cancer in groins without clinically palpable adenopathy. ${ }^{24}$ Some groups combine 
Leveridge et al.

ultrasound with fine-needle aspiration in assessment of both clinically negative and positive groins.? Magnetic resonance imaging with lymphotropic nanoparticle enhancement is an emerging technology that has been explored in penile cancer patients as well as in prostate cancer. ${ }^{25,26}$ Positron emission tomography combined with CT has shown promise in early series, with high sensitivity and specificity in the detection of occult nodal metastases. $^{27}$

The consistent and stepwise lymphatic drainage of the penis and the rarity of metastatic disease "skipping" sites on that path has led to the use of superficial node dissection in patients with clinically negative groins. The nodal package bounded by the sartorius muscle, adductor longus muscle and inguinal ligament are taken without removal of any deep nodes. Frozensection analysis of these node packages at the time of surgery allows determination of metastatic disease while attempting to minimize morbidity from the lymphadenectomy. ${ }^{3}$ Groins that show metastatic disease are subject to modified or radical lymph node dissection.

\section{Sentinel node biopsy}

The notion of effective detection of metastasis (and therefore selection of patients who would benefit from lymph node dissection) by identifying and sampling the first lymphatic landing site has long been explored. Advances in breast cancer and melanoma, along with the predictable sequence of penile lymphatic drainage have led this thrust. Cabañas ${ }^{28}$ is credited with the original description of sentinel lymph node dissection, exploiting a predictable nodal landing site superomedial to the sapheno-femoral junction.

This technique was expanded at the MD Anderson Cancer Center to include dissection of all superficial nodes medial to the saphenous vein and superior to the superficial external pudendal vein..$^{29}$ Even with this modification, the false-negative rate was $25 \%$. Horenblas and colleagues ${ }^{30}$ at the Netherlands Cancer Institute adapted dynamic sentinel node biopsy (DSNB) techniques for use in carcinoma of the penis and first published their results in 2000. They performed lymphoscintigraphy with technetium $99 \mathrm{~m}$ nanocolloid injected into the dermis around the primary tumour in 55 patients with stage $\mathrm{T} 2$ or greater disease with clini- cally negative groins on the day before surgery and injected patent blue dye on the day of surgery. The combination of the dye for lymphatic tracking and node colouring with a gamma detection probe for detection of the sentinel node allowed for determination of the first landing site of the lymphatic drainage of the tumour. This node, when identified, was resected, and pathological analysis performed. Full node dissection was undertaken 2 weeks later in the event of a positive sentinel node. Two of 24 patients with negative sentinel node biopsies subsequently presented with metastatic disease to the inguinal nodes. Several other centres have since published sentinel node series, with false-negative rates of $11 \%-29 \%$. $^{31-33}$

Two recent studies involved sentinel node biopsy concomitant with groin dissection, an approach that allowed for pathological assessment of falsenegative rates. Thirty-one such patients were recently reviewed at the MD Anderson Cancer Center. ${ }^{32}$ Two of 7 groins that harboured metastatic disease had negative sentinel node biopsies. Perdona and colleagues ${ }^{33}$ compared groups of clinically node-negative patients with stage T2-T3 penile cancer who underwent either radical lymph node dissection alone or DSNB followed by groin dissection. They achieved a false-negative rate of $11 \%$ and confirmed the utility of combining lymphoscintigraphy with intraoperative lymphatic mapping with blue dye, as $23 \%$ of "hot" nodes did not demonstrate dye uptake. Hadway and colleagues $^{34}$ from London, UK, published the first British series of DSNB for penile cancer. They performed ultrasound and fine-needle aspiration on each patient on the day of surgery and noted a falsenegative rate for fine-needle aspiration of over $50 \%$ in those groins that ultimately harboured sentinel node metastasis. Their false-negative rate for DSNB at a median follow-up of 11 months was $5 \%$, as only 1 patient with a negative sentinel node biopsy had subsequently developed nodal metastases.

The major limitation of assessing inguinal node status via sentinel node biopsy has been the high rate of false-negative groins and this has led most centres to note that a more extensive dissection remains the standard of care. The group at the Netherlands Cancer Institute has since taken measures to try to decrease the false-negative rate of DSNB by altering its protocol. ${ }^{35}$ Rigorous serial sectioning of sentinel nodes with immunohistochemical analysis is now routinely undertaken, as 
some small metastatic deposits were not found on prior sectioning protocols. They noted also that cancer was being found in groins where no sentinel node was found and have proposed full dissection for these groins based on the idea that a node infiltrated with cancer may no longer function as a drainage node and thus the lymph drainage is diverted either to an adjacent node or to the contralateral groin. Preoperative highresolution ultrasound is now used in all patients there and has shown efficacy in detecting smallvolume metastatic deposits, confirmed by fineneedle aspiration. These patients would then undergo radical lymphadenectomy. Results of these modifications in a cohort of 58 patients have recently been published, with a reduction of the false-negative rate to $4.8 \% .^{36}$ It has been noted, however, that there is likely a long learning curve to the procedure of about 20 cases (troublesome in a condition with such a low prevalence) and that the radiological and pathological expertise required are likely to be available only in large and experienced centres. ${ }^{37}$ This may have an impact on the adoption and use of DSNB in Canada, where the accrual of a high-volume case series would be expected to take a very long time for any single centre. This may expose patients to the risk of false-negative DSNB interrogation, with the resultant potential for decreased survival in late disease discovery.

\section{Risk stratification in penile cancer}

Of course, one strategy to reduce the morbidity of lymph node dissection would be to obviate the procedure by predicting those patients in whom nodal metastases are not likely to occur. This relates to the prognostic implications of the biology of the primary tumour. Several studies in penile cancer patients have demonstrated that the grade and stage of the penile primary as well as the microscopic presence of vascular invasion are the most important prognostic variables. ${ }^{38-42}$ Recent work has suggested that lymphatic invasion and an infiltrating (rather than pushing) pattern of tumour invasion are also independent prognostic factors in the development of nodal metastases. ${ }^{10,43}$ These studies have been used to stratify patients into low-risk, intermediate-risk and high-risk groups based on their primary disease pathology ${ }^{38,44}$ Solsona and colleagues ${ }^{38}$ found lowrisk patients to be those with $\mathrm{Ta}$, Tis or grade 1 dis- ease, associated with less than a $17 \%$ of nodal micrometastases. High-risk patients have stage T2 or higher disease with grade 2 or 3 disease, associated with a $68 \%-73 \%$ risk of node-positive disease. This stratification has been adopted by the European Association of Urology in its guidelines on penile cancer. ${ }^{1}$ Hungerhuber and colleagues ${ }^{44}$ stratified low-risk patients (8\% risk of occult metastasis) as those with stage $\mathrm{T} 1$ tumours and grades 1 or 2 , while high-risk patients $(75 \%$ risk of occult metastasis) were those with any grade 3 cancer. All others were in an intermediate-risk group, with a 29\% risk of metastatic disease to nodes. Ficarra and colleagues ${ }^{45}$ have recently published a nomogram for predicting the likelihood of nodal metastasis using tumour thickness, growth pattern, grade, lymphovascular invasion, corpus cavernosum invasion, corpus spongiosum invasion, urethral infiltration and clinical node status. Prospective external validation will further delineate its ultimate utility.

\section{Timing of lymphadenectomy}

Another strategy aimed at performing lymphadenectomy only in those who require it has been to defer surgery and enter patients into an active surveillance protocol, operating at the advent of clinically palpable adenopathy. This has been supported by the fact that most patients will not develop lymph node metastases. The risk and concern, of course, would be the trade-off of unnecessary and morbid surgery for potentially increased risk of failure in treating more advanced disease. Several studies have addressed this issue..$^{22,23,40,46}$ Kroon and colleagues ${ }^{22}$ recently reviewed 40 men with stage T2-T3 squamous cell carcinoma of the penis and confirmed inguinal metastasis who underwent immediate $(n=20)$ or delayed $(n=20)$ lymphadenectomy. Survival at 3 years from the date of primary tumour excision was $84 \%$ in the immediate surgery cohort, and $35 \%$ in those men who underwent node dissection after delayed presentation of clinically positive groin disease. This is in keeping with prior results and has swayed opinion in favour of early resection in patients at intermediate or high risk as previously defined.

\section{Conclusion}

There is consensus that the ultimate determination 
Leveridge et al.

of survival in patients presenting with penile cancer rests with the detection and complete resection of nodal metastases. This resection can be curative even in advanced disease, but carries with it a significant burden of morbidity that has led the thrust to develop protocols and techniques to maximize detection and minimize morbidity and unnecessary surgery. The surgical mandate in clinically positive groins is clear, as durable survival can be achieved with complete resection in some patients. The management of the clinically negative groin is less clear. The issue of assessing the likelihood of lymph node metastasis in an effort to maximize the effectiveness of penile cancer treatment while minimizing morbidity and the performance of unnecessary lymph node dissections is complex. Two disparate approaches have evolved: the first seeks to optimize detection of disease in the groin itself via minimally invasive sentinel node sampling techniques, and the other ventures to predict nodal disease based on the pathology of the primary tumour. To determine where the best results will come from will require further research, and whether these tools can be further refined or combined is as yet unclear. The low incidence of penile cancer in western nations deals a crippling blow to the prospect of large, prospective trials to best elucidate the optimal strategy, but rigorous documentation and experimentation by several centres forges ahead to improve therapy for this often virulent cancer.

From the *Department of Urology, Queen's University, Kingston, Ont., and the †Division of Urology, University of Ottawa, Ottawa, Ont.

This article has been peer reviewed.

Competing interests: None declared.

\section{References}

1. Solsona E, Algaba F, Horenblas S, et al.; European Association of Urology. EAU guidelines on penile cancer. Eur Urol 2004;46:1-8.

2. Horenblas S. Lymphadenectomy for squamous cell carcinoma of the penis. Part 2: the role and technique of lymph node dissection. BJU Int 2001;88:473-83.

3. Pettaway CA, Lynch DF, Davis JW. Tumors of the penis. In: Wein AJ, Kavoussi LR, Novick AC, et al., editors. Campbell-Walsh urology. 9th ed. Philadelphia: Saunders/Elsevier; 2007.

4. Dewire D, Lepor H. Anatomic considerations of the penis and its lymphatic drainage. Urol Clin North Am 1992;19:211-9.

5. Lont AP, Kroon BK, Gallee MPW, et al. Pelvic lymph node dissection for penile carcinoma: extent of inguinal lymph node involvement as an indicator for pelvic lymph node involvement and survival. J Urol 2007;177:947-52.

6. Culkin DJ, Beer TM. Advanced penile carcinoma. J Urol 2003;170:359-65.
7. Horenblas S. Lymphadenectomy for squamous cell carcinoma of the penis. Part 1: diagnosis of lymph node metastasis. BJU Int 2001;88:467-72.

8. Kroon BK, Valdes Olmos RA, van Tinteren H, et al. Reproducibility of lymphoscintigraphy for lymphatic mapping in patients with penile cancer. J Urol 2005;174:2214-7.

9. Kattan MW, Ficarra V, Artibani W, et al. Nomogram predictive of cancer specific survival in patients undergoing partial or total amputation for squamous cell carcinoma of the penis. J Urol 2006;175:2103-8.

10. Guimaraes GC, Lopes A, Campos RSM, et al. Front pattern of invasion in squamous cell carcinoma of the penis: new prognostic factor for predicting risk of lymph node metastases. Urology 2006;68:148-53.

11. Horenblas S, van Tinteren H, Delemarre JF, et al. Squamous cell carcinoma of the penis: accuracy of tumour, nodes and metastasis classification system, and role of lymphangiography, computerized tomography scan and fine needle aspiration cytology. J Urol 1991;146:1279-83.

12. Jacobellis U. Modified radical inguinal lymphadenectomy for carcinoma of the penis: technique and results. J Urol 2003;169:1349-52.

13. Bevan-Thomas R, Slaton JW, Pettaway CA. Contemporary morbidity from lymphadenectomy for penile squamous cell carcinoma: the M.D. Anderson Cancer Center experience. J Urol 2002;167:1638-42.

14. Catalona WJ. Modified inguinal lymphadenectomy for carcinoma of the penis with preservation of saphenous veins: technique and preliminary results. J Urol 1988; 140:306-10.

15. Bouchot 0 , Rigaud J, Maillet $\mathrm{F}$, et al. Morbidity of inguinal lymphadenectomy for invasive penile carcinoma. Eur Urol 2004:45:761-6.

16. Nelson BA, Cookson MS, Smith JA Jr, et al. Complication of inguinal and pelvic lymphadenctomy for squamous cell carcinoma of the penis: a contemporary series. J Urol 2004;172:494-7.

17. Tobias-Machado M, Tavares A, Ornellas AA, et al. Video endoscopic inguinal lymphadenectomy: a new minimally invasive procedure for radical management of inguinal nodes in patients with penile squamous cell carcinoma. J Urol 2007;177:953-8.

18. Haas GP, Blumenstein BA, Gagliano RG, et al. Cisplatin, methotrexate and bleomycin for the treatment of carcinoma of the penis: a Southwest Oncology Group study. J Urol 1999;161:1823-5.

19. Theodore C, Skoneczna I, Bodrogi I, et al.; EORTC Genito-Urinary Tract Cancer Group. A phase II multicentre study of irinotecan (CPT 11) in combination with cisplatin (CDDP) in metastatic or locally advanced penile carcinoma (EORTC PROTOCOL 30992). Ann Oncol 2008; 19:1304-7.

20. Leijte JAP, Kerst JM, Bais E, et al. Neoadjuvant chemotherapy in advanced penile carcinoma. Eur Urol 2007;52:488-94.

21. Bermejo C, Busby JE, Spiess PE, et al. Neoadiuvant chemotherapy followed by aggressive surgical consolidation for metastatic penile squamous cell carcinoma. J Urol 2007; 177:1335-8.

22. Kroon BK, Horenblas A, Lont AP, et al. Patients with penile carcinoma benefit from immediate resection of clinically occult lymph node metastases. J Urol 2005; 173:816-9.

23. Ornellas AA, Correia Seixas AL, Marota A, et al. Surgical treatment of invasive squamous cell carcinoma of the penis: retrospective analysis of 350 cases. J Urol 1994; 151:1244-9.

24. Hall TB, Barton DP, Trott PA, et al. The role of ultrasound-guided cytology of groin lymph nodes in the management of squamous cell carcinoma of the vulva: 5-year experience in 44 patients. Clin Radiol 2003;58:367-71.

25. Harisinghani MG, Barentsz J, Hahn PF, et al. Noninvasive detection of clinically occult lymph-node metastases in prostate cancer. N Engl J Med 2003;348:2491-9.

26. Tabatabaei S, Harisinghani M, McDougal WS. Regional lymph node staging using lymphotropic nanoparticle enhanced magnetic resonance imaging with ferumoxtran-10 in patients with penile cancer. J Urol 2005;174:923.

27. Scher $B$, Seitz $M$, Reiser $M$, et al. 18F-FDG PET/CT for staging of penile cancer. J Nucl Med 2005;46:1460-5.

28. Cabañas RM. An approach for the treatment of penile carcinoma. Cancer 1977;39: 456-66.

29. Pettaway CA, Pisters LL, Dinney PN, et al. Sentinel lymph node dissection for penile carcinoma: the MD Anderson Cancer Center experience. J Urol 1995;154:1999-2003.

30. Horenblas $S$, Jansen L, Meinhardt $W$, et al. Detection of occult metastasis in squamous 
cell carcinoma of the penis using a dynamic sentinel node procedure. J Urol 2000;163: 100-4.

31. Tanis PJ, Lont AP, Meinhardt W, et al. Dynamic sentinel node biopsy for penile cancer: reliability of a staging technique. J Urol 2002;168:76-80.

32. Spiess PE, Izawa Jl, Bassett R, et al. Preoperative lymphoscintigraphy and dynamic sentinel node biopsy for staging penile cancer: results with pathological correlation. J Urol 2007;177:2157-61.

33. Perdona S, Autorino R, de Sio M, et al. Dynamic sentinel node biopsy in clinically nodenegative penile cancer versus radical inguinal lymphadenectomy: a comparative study. Urology 2005;66:1282-6.

34. Hadway P, Smith Y, Corbishley C, et al. Evaluation of dynamic lymphoscintigraphy and sentinel lymph-node biopsy for detecting occult metastases in patients with penile squamous cell carcinoma. BJU Int 2007; 100:561-5.

35. Kroon BK, Horenblas S, Estourgie SH, et al. How to avoid false-negative dynamic sentinel node procedures in penile carcinoma. J Urol 2004;171:2191-4.

36. Leilte JAP, Kroon BK, Valdés Olmos RA, et al. Reliability and safety of current dynamic sentinel node biopsy forpenile carcinoma. Eur Urol 2007;52:170-7.

37. Ficarra V, Galfano A. Should the Dynamic Sentinel Node Biopsy (DSNB) be considered the gold standard in the evaluation of lymph node status in patients with penile carcinoma? Eur Urol 2007;52:17-21.

38. Solsona E, lborra I, Rubio J, et al. Prospective validation of the association of local tumor stage and grade as a predictive factor for occult lymph node micrometastasis inpatients with penile carcinoma and clinically negative inguinal lymph nodes. J Urol 2001; 165:1506-9.

39. Slaton JW, Morgenstern N, Levy DA, et al. Tumor stage, vascular invasion and the percentage of poorly differentiated cancer: independent prognosticators for inguinal lymph node metastasis in penile squamous cancer. J Urol 2001;165:1138-42.

40. McDougal WS. Carcinoma of the penis: improved survival by early regional lymphadenectomy based on the histological grade and depth of invasion of the primary lesion. I Urol 1995; 154:1364-6.

41. Villavicencio H, Rubio-Briones J, Regalado R, et al. Grade, local stage and growth pattern as prognostic factors in carcinoma of the penis. Eur Urol 1997;32:442-7.

42. Theodorescu D, Russo P, Zhang ZF, et al. Outcomes of initial surveillance of invasive squamous cell carcinoma of the penis and negative nodes. J Urol 1996;155:1626-31.

43. Ficarra V, Zattoni F, Cunico SC, et al. Artibani W for the North-Eastern Uro-Oncological Group Penile Cancer Project. Lymphatic and vascular embolizations are independent predictive variables of inguinal lymph node involvement in patients with squamous cell carcinoma of the penis. Cancer 2005; 103:2507-16.

44. Hungerhuber E, Schlenker B, Karl A, et al. Risk stratification in penile carcinoma: 25year experience with surgical inguinal lymph node staging. Urology 2006;68:621-5.

45. Ficarra $V$, Zattoni F, Artinai W, et al. Nomogram predictive of pathological inguinal lymph node involvement in patients with squamous cell carcinoma of the penis. J Urol 2006;175:1700-5.

46. McDougal WS, Kirchner FK Jr, Edwards RH, et al. Treatment of carcinoma of the penis: the case for primary lymphadenectomy. J Urol 1986;136:38-41.

Correspondence: Dr. D. Robert Siemens, Empire 4, Kingston General Hospital, 76 Stuart St., Kingston ON K7L 2V7; siemensr@kgh.kari.net

CUAJ is read by at least 3000 urologists and related specialists in Canada and around the world.

If you want to reach urologists, there is no better place for your classified advertisement. Please contact the journal at journal@cua.org or call 514 395-0376 ext. 40 for a price list, specifications and deadlines. 